\title{
An acquisition and analysis system for on-line experiments on the neurophysiology of learning
}

\author{
R. A. ROEMER, C. F. CEGAVSKE, and R. F. THOMPSON \\ Harvard University, Cambridge, Massachusetts 02138 \\ and \\ M. M. PATTERSON \\ Kirksville College of Osteopathic Medicine, Kirksville, Missouri 69501
}

\begin{abstract}
An integrated hardware and software system is used in studying neural correlates of learning. The hardware system is designed to maximize experimental flexibility, while the software system is designed to maximize computer speed for data acquisition and programmer efficiency for data analysis.
\end{abstract}

The dat al acquisition and analysis system described here is a hybrid hardware and softuare contiguration used in the study of neural correlates of behavioral plasticity. The hardware consists of a data switching pancl: signal conditioning circuitry; a PDP-12 computer with $12 \mathrm{~K}$ of memory, LINC tapes, and RK8 disk: photographic equipment: and an $x-y$ plotter. The soltualre system consists of assembly language programs for data acquisition. preliminary analysis. and mass storage; and interpreter language programs for more extensive analysis of data.

Data acquisition is more efticiently done, in terms of computer timee. in assembly language. Such programs are more quickly executed and are not constrained by conventions of languages that are generally designed to facilitate programming and not necessalrily program execution. We have several types of acquisition progtams. all of which are designed to optimize one form of neurophysiological datat acculisition or another, for the different investigators in our laboratory. The program discussed here is employed during the classical conditioning of the rabbit's nictitating membrane (NM) response. In this case, an analog record of the membrane response observed during a trial is recorded on disk. In addition, average time histograms of multiple unit activity and the associated averaged evoked potentials are obtained from two electrodes and stored on the disk for later, more detailed data analysis. Quantitative analysis of these data is accomplished in FOCAL-12. Qualitative and descriptive analyses are

The work was supported in part by Research Grants NS 07661 (R.F.T.) from NINDS and GB 40147X (R.F.T.) from NSF R.A.R. s current address: Eastern Pennsylvania Psychiatric Institute, Philadelphia, Pennsylvania 19129. C.F.C.'s and R.F.T.'s current address: Department of Psychobiology. University of California, Irvine, Calitornia 92664 performed by assembly language programs for pseudo llire-dimensional plotting of successive response measures over trials and for the production of motion pictures to portray the dynamics of these responses wer the session.

\section{THE HARDWARE CONFIGURATION}

The hardware configuration consists of two 10-ft free-standing equipment consoles. One console houses the computer, extended memory, LINC tape, and RK8 disk. The other console contains the laboralery switching panel, high-and low-pass filters, attenuators. 12 Schmitt triggers, an oscilloscope, an $x-y$ ploflet low-frequency function generator, a wide-range uscillator, and a Sony quadraphonic tape recorder wilh a Vetter FM adapter for one channel. Except lon the oscilloscope, all of these items and all of the input ouput channels of the computer are hardin ired lo a MAC panel receiver. In addition to the rabbil room, all other experimental rooms in the laboratory have at least 20 hardwired data lines to this suitching panel. Any standard experimental contiguration can then be obtained by merely placing an appropriately wired patch panel in the receiver. Within our laboratory, this flexibility is an asset, since we alle studying the neural correlates of plasticity and learning on several physiological levels. These studies lange from intact chronic preparations to model system investigations of synaptic effects during habituation This suitching option reduces to a minimum the time required to recontigure from one experimental procedure to another.

\section{THE SOFTWARE CONFIGURATION}

Our software system, which operates under DI $\Lambda$ L-MS. tries to maximize computer efficiency 
during data acquisition and programmer efficiency during data analysis. All acquisition software is "ritten in LAP6-DIAL and stores data in formalized tile structures on LINC tape or disk. While some preliminary analyses are performed by the assembly language programs, the major analyses are performed by FOCAL-12 programs written by each individual investigator. FOCAL-12, while computationally slow, is very simple to learn and use. Simplicity, in this case, is a valuable asset, since our primary interest is neurophysiology and not the intricacies of computer programming per se. Several other analysis programs that are useful to all investigators have been written in assembly language and generally deal with graphic displays of data preserved on tape or disk, or the reformatting of such data.

\section{NEURAL AND BEHAVIORAL DATA ACQUISITION DURING CLASSICAL CONDITIONING}

The computer program described here is designed to acquire records of neural activity observed during a classical conditioning paradigm. The specific procedure employed is the tone-airpuff training of a rabbit's nictitating membrane response. Multipleunit and evoked potential data from two electrodes are collected during three contiguous time periods of approximately $250 \mathrm{msec}$ each: pre-CS, CS, and UCS periods. A fifth record is obtained from the analog signal generated by the movement of the rabbit's nictitating membrane, again recorded during the three time periods. A sixth record acquires the onset time of both the CS and US. This program, written in LAP6-DIAL, operates in conjunction with the comparator one-shot multivibrator system described by Cegavske, Roemer, and McCurnin (1973).

In terms of computing time, we have found it efficient to use the comparator system to preprocess multiple-unit data by quantizing these data as digital events in time. The computer then counts these events as a function of time. For each trial, the program calculates the cumulative sum of unit-time histograms for each electrode and the average evoked potential associated with each electrode. When a specified number of samples have been taken, these averages and sums are saved on disk storage for later off-line analysis. In addition, the NM record associated with each trial is written to disk.

During paired trials, we employ a total of nine trials per "block," where eight trials are CS-UCS pairings and the ninth trial is a CS-alone, probe, trial. Under the unpaired procedure, a total of 16 trials is used per block, where eight trials are US-alone presentations randomized with eight CS-alone presentations. A CS-alone trial is indicated to the computer by a grounded signal on one of the sense lines (13). During initialization, the computer program adjusts the scale factors used in calculating evoked potential averages to the appropriate values. During a paired session,
Table 1

Decimal Fractions Up to $1 / 33$ (on Right) and the Corresponding Octal Values for a 12-Bit Fixed-Point Fraction Scaled at -0*

\begin{tabular}{llllll}
\hline 3777 & 1 & 0252 & $1 / 12$ & 0131 & $1 / 23$ \\
2000 & $1 / 2$ & 0234 & $1 / 13$ & 0125 & $1 / 24$ \\
1252 & $1 / 3$ & 0222 & $1 / 14$ & 0122 & $1 / 25$ \\
1000 & $1 / 4$ & 0213 & $1 / 15$ & 0116 & $1 / 26$ \\
0631 & $1 / 5$ & 0200 & $1 / 16$ & 0113 & $1 / 27$ \\
0525 & $1 / 6$ & 0170 & $1 / 17$ & 0111 & $1 / 28$ \\
0447 & $1 / 7$ & 0162 & $1 / 18$ & 0106 & $1 / 29$ \\
0400 & $1 / 8$ & 0154 & $1 / 19$ & 0104 & $1 / 30$ \\
0343 & $1 / 9$ & 0146 & $1 / 20$ & 0104 & $1 / 31$ \\
0314 & $1 / 10$ & 0141 & $1 / 21$ & 0100 & $1 / 32$ \\
0272 & $1 / 11$ & 0135 & $1 / 22$ & 0076 & $1 / 33$ \\
\hline
\end{tabular}

*Therefore, the binary point lies between the sign bit and the leftmost bit.

117 trials are presented (104 CS-US pairings and 13 CS-alone probes); during unpaired sessions, a total of 208 trials are presented (104 US alone and 104 CS alone). This results in 13 sets or blocks of data.

\section{AVERAGING METHODS}

An earlier version of this program used a "shift and add" strategy to calculate the averages. In this case, since each logical shift of one place to the right of a binary word is an effective division by two, an average with eight samples can be developed as follows: For each sample, shift right three places (this is an effective division by eight), then add the resultant to the cumulative average. The disadvantage of the method is that the averaged waveform appears to "grow" in amplitude. A later version uses the fractional multiple capability of the PDP-12 to calculate an average which does not "grow" in anılitude.

It can be shown that solving Equation 1 results in such an average.

$$
\bar{X}_{N}=\bar{X}_{N-1}+\frac{\bar{X}(N-1)+X i}{N} .
$$

The fractional coefficients for a 12-bit fractional fixed point computer such as the PDP-12 are listed in Table 1. These coefficients have been appropriately rescaled to maintain the proper scale factor.

\section{SEQUENCE OF OPERATION}

During the interval between periods of data acquisition, the program can display the summed histograms of unit activity, the accumulated average evoked responses, the immediately preceding NM response, and the onset time of the CS and the US. When a pre-CS start signal occurs, it interrupts the display routine and initializes the acquisition portion of the program.

A 3-nisec "tick" rate is established for the real-time 
clock; interrupting is enabled for Interrupt Inputs 2 and 3 (Neural Multiple Units 1 and 2); pointers are set for autcindexing of data storage; and a run-length clock of 256 clock ticks $(768 \mathrm{msec})$ is enabled. The program then loops until one of tour events occurs: (1) The 3-msec clock ticks. (2) A multiple-unit discharge triggers Input 2 and/or Input 3 , (3) a CS event is detected on Sensc Line 0. and (4) a UCS event is detected on Sense Linc 1.

If a clock tick occurs, analog-to-digital conversion occurs on Channels 10 (FM), 11 , and 12 (11 and 12 carry evoked potential data for the two electrodes), and the resultants are retained in three buffers, and the data storage index pointers are advanced. The magnitude of the NM signal on Analog Channel 10 is compared to a criterion level selected via a potentiometer on the computer. If the criterion level is exceeded during the pre-CS period, data acquisition for the trial is aborted, and $\mathrm{K} 4$ is closed for $2 \mathrm{sec}$, and all the temporary buffers are cleared.

If a multiple-unit event is detected on Input 2 and/or Input 3, a buffer memory location is incremented as a function of the elapsed time.

If the onset of a CS or UCS event is detected, a flag indicating the time at which these occurred is set. The entire looping sequence continues until the run-length clock has expired.

When the run-length clock expires, the NM response record is written to disk. The program then prints out on the Teletype (1) the trial number; (2) if the trial was a CS-alone trial; (3) the time of the CS onset; (4) the time of the UCS onset: (5) the criterion/abort level selected by Analog Channel 7; and (6) the latency of the onset of the NM response as determined by the NM response exceeding that criterion level during the CS or UCS period. The latter allows us to determine if a conditioned response occurred.

After the printout is completed, all the averages and unit-time histograms are updated, the bulfers are cleared, and the display subroutine is resumed, with interrupting enabled for the next pre-CS signal.

After the specitied number of trials per average have been run, the histograms, averages, and the CS and UCS flags are written onto successive disk locations. $l t$ is these records which are then subjected to oftline analysis using FOCAL-12.

\section{MULTIPLE-UNIT ANALYSIS USING FOCAL-12}

Multiple unit data from the 13 sets or blocks of experimental data (paired, unpaired CS alone, unpaired US alone) are analyzed in the three time periods: pre-CS, CS, and US. The pre-CS period starts with trial onset and runs for $240 \mathrm{msec}$, ending a few milliseconds before CS onset. The CS period is detined as the $240 \mathrm{msec}$ prior to US onset. A period of a few milliseconds between the pre-CS and CS periods is not analyzed. The US period begins with US onset and runs for $240 \mathrm{msec}$.

Within each $240-\mathrm{msec}$ time period, the multiple-unit activity is totalled and divided by 8 , giving mean total activity for each period. Recall that each histogram is the sum of eight observations or trials. After each block of data in the experimental session has been treated in this way, a grand mean and a standard deviation are computed from the mean total activity scores of the pre-CS periods. Standardized scores are then calculated according to the following formula for each pre-CS block (i).

$$
\mathrm{S}_{\mathrm{i}}=\frac{(\text { Pre-CS mean total activity })_{\mathrm{i}}-\text { Grand mean }}{\text { Pre-CS Standard Deviation }} .
$$

Similarly, standardized scores for the CS and US periods were calculated according to the following formula:

$\mathrm{SS}_{\mathrm{i}}=$

(CS

(US mean total activity) $)_{\mathrm{i}}$-(Pre-CS mean total activity $)_{\mathrm{i}}$

Pre-CS Standard Deviation

The Teletype then prints these scores for the three time periods wer the whole experimental session. The analysis allows the following to be determined: (1) If a change in the amount of background (control) multiple-unit activity has occurred over the training session. (2) If a change in the amount of activity has occurred as a result of $C S$ or US presentation. (3) The magnitude of that change in terms of the standard deviation of the control periods.

Multiple-unit data from probe trials are handled similarly except, instead of mean total activity for an eight-trial block. total activity from each of the three different time periods of individual trials is used.

Figure 1 illustrates the results of these computations for one hippocanipal electrode. Figure 1 A shows, for the 13 hlocks of pre-CS records, the subject scores associated with each block. Figures $1 B$ and $1 C$ present the standardized scores of the CS period. In this case. the $C S$ period has been analyzed in two time periods: multiple-unit activity during the first and second half of the CS presentation.

Figures $(1)$ and $1 E$ show standardized scores of the multiple-unit activity during extinction for the period which was previously the US period. This time is again analyzed in tuo parts: first and second half of the time period.

Another form of graphic analysis is presented in Figure 2. Here are plotted the 13 averaged NM responses $(A)$ during a paired conditioning session. Figures $2 \mathrm{~B}$ and 2C display, respectively, time histograms of all units whose amplitude exceeded $5.5 \mu \mathrm{V}$ and those whose amplitude exceeded $70 \mu \mathrm{V}$. 

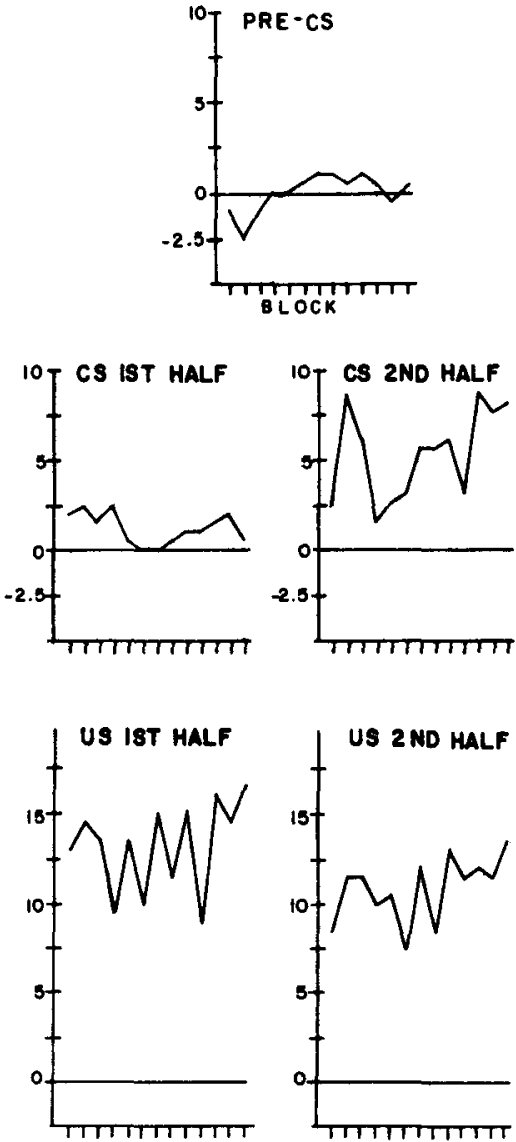

Figure 1. Subject score and standardized score transformations of multiple-unit activity recor ding during 13 consecutive blocks of eight trials. The pre-CS curve represents subject scores. The $\mathrm{CS}$ and US curves are standardized scores based on the pre-CS values for each block (see text). First and second half in each case refers to the first and second half of the respective stimulus periods.

Note the systematic development of behavioral CRs in Figure $2 \mathrm{~A}$ and the corresponding increase in the histogram of Figure $2 \mathrm{C}$. The electrode in this case was in the abducens nucleus, which is the final neural output pathway that controls the NM response.

\section{NICTITATING MEMBRANE [NM] RESPONSE ANAL YSIS USING FOCAL-12}

Each individual NM response is analyzed for its onset latency, peak latency, peak amplitude, and the area under the curve. The values stored in each subsequent 3 -msec interval is tested to determine if it is greater than that produced by $0.5 \mathrm{~mm}$ of NM closure. The first interval found to meet the criterion is defined as the onset latency of the response. Responses occurring before US onset are considered conditioned responses on paired trials. Figure 3 presents a comparison of a typical NM response recorded on the polygraph $(\mathrm{C})$ and a plot of the same response recorded by the computer (D). Each NM response is analyzed for 240 msec on each side of US onset, and the "integral" of the response is calculated for both periods. In cases where the NM was closed more than once during the same 240-msec period, the "integral" is calculated for each response, and a total "integral" is also calculated for the period.

The Teletype then prints the onset latency, the peak latency, the peak amplitude, the "area" under the response in the CS period, and the "area" under the response in the US period.

\section{VALIDATION OF PROGRAMS}

\section{Scoring the NM Response for CRs}

One of the often overlooked aspects of computer systems is determining whether or not that which is analyzed is that which you think you analyzed. This, of course, is the issue of program validation. It was dealt with, in the case of the NM analysis, by a side-by-side comparison of two methods of analysis. The traditional method of manual scoring of the NM responses by one of the authors, who has had long experience in scoring such data (M.M.P.), was compared to the second method, which has just been described. In a total of 504 trials taken from two animals on four training sessions, there were 10 discrepancies found in determining a conditioned response. Six of these resulted from the computer's using a slightly lower threshold than the experimenter, so that small
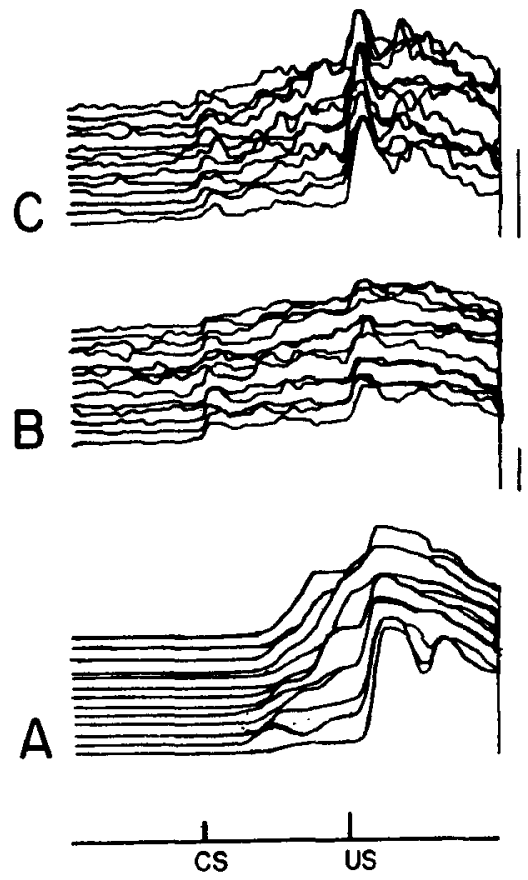

Figure 2. Time histograms of the average nictitating membrane response (A), abducens nucleus multiple units with amplitudes greater than $55 \mu \mathrm{V}$ (B), and abducens nucleus multiple units with amplitudes greater than $70 \mu \mathrm{V}$ (C) for 13 consecutive averages of eight trials. Abscissa $=768 \mathrm{msec}$, with $\mathrm{CS}$ and US onsets indicated. Ordinate scale in arbitrary units. 


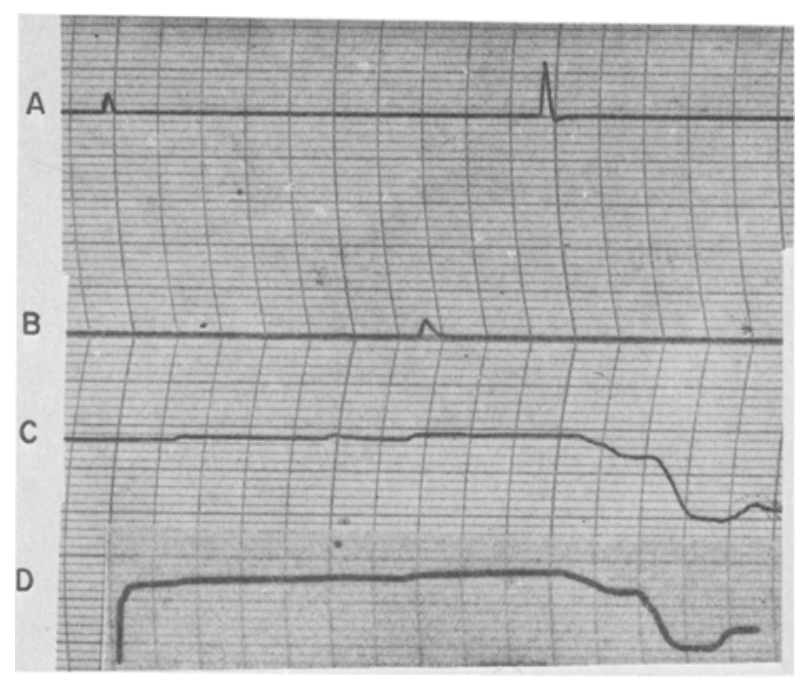

Figure 3. Polygraph and computer records of a nictitating membrane (NM) response to an air puff. (A) Pre-CS and US markers, (B) timing mark, (C) polygraph record of NM responses, (D) computer plot. Time is $\mathbf{5 0} \mathbf{m s e c}$ division.

responses which did not quite reach the experimenter"s threshold were counted by the computer. The other four resulted from a difference in instructions to the computer and to the experimenter. The computer was programmed to determine a CR by the latency of the response at the point it met the criterion for detecting a response. The experimenter decided that a response was a $C R$ by the onset latency of the response, i.e., where the first change from baseline could be detected. In the four later instances, the US onset fell between the time of this first detectable change and the time when the response met the amplitude criterion. By the first-detectable change method. the response was a CR; by the amplitude criterion method, it was not.

In summary, the experimental system described here is designed to maximize various types of efficiency. During experimentation, computational efticiency is maximized, while during data analysis, the experimenter's time is maximized. The use of patch pancls to reconfigure the laboratory for various experinents is designed to further enhance the efficiency of the scientist.

The hardware and software system described here is a central instrument in our studies of learning. As such. it and similar systems provide some major advantages over other approaches to these problems. The tirst is experimental flexibility, which permits a wide range of investigations to be conducted with a minimum of time being lost in reconfiguring for a given experiment. A second major advantage is in the integrity of the data which are collected. The machine does not change its operating criteria due to fatigue. The high reliability of the acquisition and analyses is a significant issue. A third advantage is that the presence of a computer system in an experiment brings with it a unicue form of experimental rigor.

\section{REFERENCE}

Cegayske, C. F.. Roemer, R. A., \& McCurnin, T. W. A low-cosl integrated circuit comparator system. Behavior Research Methods \& Instrumentation, 1973, 5, 428-430. 